\title{
A stable and accurate relaxation technique using multiple penalty terms in space and time
}

Hannes Frenander and J an Nordström

The self-archived version of this journal article is available at Linköping University Electronic Press:

http:/ / urn.kb.se/ resolve?urn=urn:nbn:se:liu:diva- 139885

N.B.: When citing this work, cite the original publication.

Frenander, H., Nordström, J ., (2017), A stable and accurate relaxation technique using multiple penalty terms in space and time, Dynamics of atmospheres and oceans (Print), 79, 56-65.

https:// dx.doi.org/ 10.1016/j.dynatmoce.2017.07.003

Original publication available at:

https:// dx.doi.org/ 10.1016/j.dynatmoce.2017.07.003

Copyright: Elsevier: 24 months

http:// www.elsevier.com/

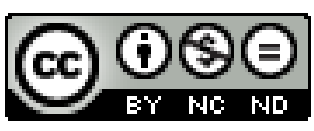




\title{
A stable and accurate data assimilation technique using multiple penalty terms in space and time
}

\author{
Hannes Frenander, Jan Nordström \\ Division of Computational Mathematics, Department of Mathematics, Linköping \\ University, SE-58183 Linköping. Sweden
}

\begin{abstract}
A new method for data assimilation based on weak imposition of external data is introduced. The technique is simple, easy to implement, and the resulting numerical scheme is unconditionally stable. Numerical experiments show that the error growth naturally present in long term simulations can be prevented by using the new technique.
\end{abstract}

Keywords: data assimilation, summation-by-parts, weak boundary conditions, multiple penalties, stability, finite differences

\section{Introduction}

In many applications in science and engineering, one wants to combine results from ongoing simulations with observations. The observations are typically made at a few positions in the spatial domain under consideration, and during limited time intervals. Consequently, numerical techniques for incorporating such data in the simulations are needed. Moreover, the additional data must improve the results by, for example, reducing the error or increasing the rate of convergence.

In the weather prediction community, many data assimilation techniques are based on minimizing the deviation from the observations by finding the minimum of a cost function. The $3 \mathrm{D}$ varational (3D-Var) and $4 \mathrm{D}$ variational (4D-Var) methods are based on this strategy [4, 5]. Another popular technique for data assimilation in geophysical problems is Newtonian Nudging, [15, 1]. In this technique, relaxation terms are added to the governing equations that force the solution towards an observed state.

URL: hannes.frenander@liu.se (Hannes Frenander, Jan Nordström) 
In this work, we introduce a similar but provable stable technique for data assimilation based on Summation-By-Parts (SBP) operators [8, 11, 16, 10, 14] and Simultaneous Approximation Terms (SAT) [2, 3]. This new technique is an extension of the Multiple Penalty Technique (MPT) introduced in [13, 6], where SAT's are implemented at grid points inside the computational domain. Besides being simple and easy to implement, the MPT always results in a provably stable scheme.

For simplicity and clarity of presentation, we consider the additional data to be exact in the main part of the paper. However, similar results would be also be obtained for inaccurate data during long time calculations; we shortly discuss this subject in Appendix A.

The previous version of the MPT required data to be known at the appropriate spatial grid points during the entire simulation. In this paper, we extend this formulation such that the MPT can be applied at grid points during limited time intervals, which models real life observations better. We show how this extension is implemented, that stability is preserved and that error growth in time is prevented when it is applied.

The rest of this paper will proceed as follows. In Section 2, we describe how to apply the MPT in time and space on a model problem, and how it can be used to reduce errors. The results are generalized to the linearized shallow water equations in Section 3. Finally, in Section 4, we summarize the results, and draw conclusions.

\section{The MPT in time and space on a model problem}

As a first example, consider the advection equation in one space dimension with periodic boundary conditions,

$$
\begin{aligned}
u_{t}+u_{x} & =0, \quad x \in[0,1], t \in[0, T], \\
u(0, t) & =u(1, t) \quad t \in[0, T], \\
u(x, 0) & =f(x) \quad x \in[0,1],
\end{aligned}
$$

where we have used subscripts to denote partial derivatives, i.e. $u_{t}=\partial u / \partial t$ and $u_{x}=\partial u / \partial x$. The function $f$ is the initial data.

Multiplying (1) with $u$ and integrating in space and time yields,

$$
\int_{0}^{1} u^{2}(x, T) d x=\int_{0}^{1} f^{2} d x .
$$


According to (2), the solution at the final time $T$ is bounded by the initial data, and the problem is therefore well-posed [17].

\subsection{The discrete problem}

To discretize (1), we use finite difference operators $D_{x}=P_{x}^{-1} Q_{x}$ and $D_{t}=P_{t}^{-1} Q_{t}$ on SBP form, where the subscript denotes the derivative which is being approximated. The matrices $P_{x}$ and $P_{t}$ are symmetric and positive definite, and the matrices $Q_{x}$ and $Q_{t}$ satisfy the SBP property, $Q_{t, x}+Q_{t, x}^{T}=$ $B_{t, x}=\operatorname{diag}(-1,0, \ldots, 0,1)$. Further, we assume that known additional data is available at a few spatial grid points during a few limited time intervals. We denote these spatial grid points and time intervals by $\Omega_{s}$ and the additional data by $g(x, t)$. The additional data will be implemented using SAT's [13, 6].

The fully discrete version of (1) including the observations becomes,

$$
\begin{array}{r}
\left(D_{t} \otimes I_{x}\right) v+\left(I_{t} \otimes D_{x}\right) v=\alpha_{t}\left(P_{t}^{-1} E_{0 t} \otimes I_{x}\right)(v-\bar{f})+ \\
\alpha_{x}\left(I_{t} \otimes P_{x}^{-1} E_{0 x}\right)\left(v_{x=0}-v_{x=1}\right)+\beta_{x}\left(I_{t} \otimes P_{x}^{-1} E_{N x}\right)\left(v_{x=1}-v_{x=0}\right)+ \\
\sum_{x_{i}, t_{j} \in \Omega_{s}} \alpha_{i j}\left(P_{t}^{-1} E_{i t} \otimes P_{x}^{-1} E_{j x}\right)\left(v-\bar{g}_{i j}\right) .
\end{array}
$$

The observations are included in the last term on the right hand side. The elements of the matrices $E_{i t, x}$ are zero, except at the element $(i, i)$, where it is equal to one. The sum includes a few positions, $x_{j}$, for a few time intervals. Furthermore, the symbol $\otimes$ denotes the Kronecker product, defined by,

$$
A \otimes B=\left[\begin{array}{cccc}
A_{11} B & \ldots & \ldots & A_{1 n} B \\
\vdots & \ddots & \ddots & \vdots \\
\vdots & \ddots & \ddots & \vdots \\
A_{m 1} B & \ldots & \ldots & A_{m n} B
\end{array}\right]
$$

for two arbitrary matrices $A$ and $B$. In (3), $v_{x=0,1}$ denotes the numerical solution at $x=0,1, \bar{g}_{i j}$ is the known data projected on a grid vector, $\bar{f}$ is the initial data and $\alpha_{x}, \alpha_{t}, \beta_{x}$ and $\alpha_{i j}$ are scalar penalty coefficients to be determined.

Applying the discrete energy method (i.e. multiplying with $v^{T}\left(P_{t} \otimes P_{x}\right)$ from the left and adding the transpose of the result) to (3) gives,

$$
\begin{array}{r}
v^{T}\left(E_{N t} \otimes P_{x}\right) v=\bar{f}^{T}\left(E_{0 t} \otimes P_{x}\right) \bar{f}-(v-\bar{f})^{T}\left(E_{0 t} \otimes P_{x}\right)(v-\bar{f})+ \\
\sum_{x_{i}, t_{j} \in \Omega_{s}} \alpha_{i j}\left|v_{i j}-\bar{g}_{i j}\right|^{2}+\alpha_{i j}\left(\left|v_{i j}\right|^{2}-\left|\bar{g}_{i j}\right|^{2}\right) .
\end{array}
$$


Here, we have used $\alpha_{x}=-\beta_{x}=-1 / 2, \alpha_{t}=-1$ and denoted $v_{i j}=\left(E_{i t} \otimes\right.$ $\left.E_{j x}\right) v$. Also, the notation $\left|v_{i j}\right|^{2}=v^{T}\left(E_{i t} \otimes E_{j x}\right) v$ is used. By choosing $\alpha_{i j} \leq 0$, the solution is bounded by the initial data $\bar{f}$, and the scheme is stable. Moreover, if $v_{i j}=\bar{g}_{i j}$ and $v=\bar{f}$ at $t=0$, (4) mimics the continous estimate (2). Note that the additional MPT terms in (4) leads to a dissipative effect, i.e. they give a negative contribution to the energy. In the next section, we will show that this is important.

\subsection{Error analysis}

To obtain an instructive error equation, we consider the corresponding semi-discrete approximation of (1),

$$
\begin{array}{r}
v_{t}+D_{x} v=-\frac{1}{2}\left(I_{t} \otimes P_{x}^{-1} E_{0 x}\right)\left(v_{x=0}-v_{x=1}\right)+ \\
\frac{1}{2}\left(I_{t} \otimes P_{x}^{-1} E_{N x}\right)\left(v_{x=1}-v_{x=0}\right)+\sum_{x_{i}, t \in \Omega_{s}} \alpha_{i} P_{x}^{-1} E_{j x}\left(v_{i}-\bar{g}_{i}\right),
\end{array}
$$

where $\bar{g}_{i}$ is the additional data and $\alpha_{i} \leq 0$ the additional penalty parameters.

Next, consider (5) with the exact solution $\bar{u}$ injected on the grid,

$$
\begin{array}{r}
\bar{u}_{t}+D_{x} \bar{u}=-\frac{1}{2}\left(I_{t} \otimes P_{x}^{-1} E_{0 x}\right)\left(\bar{u}_{x=0}-\bar{u}_{x=1}\right)+ \\
\frac{1}{2}\left(I_{t} \otimes P_{x}^{-1} E_{N x}\right)\left(\bar{u}_{x=1}-\bar{u}_{x=0}\right)+\sum_{x_{i}, t \in \Omega_{s}} \alpha_{i} P_{x}^{-1} E_{j x}\left(\bar{u}_{i}-\bar{g}_{i}\right)+T e
\end{array}
$$

where $T e$ is the truncation error. Subtracting (6) from (5) results in the error equation,

$$
\begin{array}{r}
e_{t}+D_{x} e=-\frac{1}{2} P_{x}^{-1} E_{0 x}\left(e_{0}-e_{N}\right)+\frac{1}{2} P_{x}^{-1} E_{N x}\left(e_{N}-e_{0}\right)+ \\
\sum_{x_{i}, t \in \Omega_{s}} \alpha_{i} P_{x}^{-1} E_{j x} e_{i}+T e
\end{array}
$$

where $e=v-\bar{u}$. In (7), $e_{i}$ denotes the error at grid point $i$.

Multiplying (7) by $e^{T} P_{x}$ from the left and adding the transpose of the outcome results in,

$$
\frac{\partial}{\partial t}\left(\|e\|_{P_{x}}^{2}\right)=\sum_{x_{i}, t \in \Omega_{s}} 2 \alpha_{i}\left|e_{i}\right|^{2}+2 e^{T} P_{x} T e .
$$


Using that

$$
\frac{\partial}{\partial t}\left(\|e\|_{P_{x}}^{2}\right)=2\|e\|_{P_{x}}\left(\|e\|_{P_{x}}\right)_{t}, \quad e^{T} P_{x} T e \leq 2\|e\|_{P_{x}}\|T e\|_{P_{x}},
$$

and dividing both sides of (8) with $2\|e\|_{P_{x}}$ results in,

$$
\frac{\partial}{\partial t}\left(\|e\|_{P_{x}}\right) \leq-\eta(t)\|e\|_{P_{x}}+\|T e\|_{P_{x}}^{\max },
$$

where $\|T e\|_{P_{x}}^{\max }$ denotes the upper bound of $\|T e\|_{P_{x}}$ and

$$
\eta(t)=\sum_{x_{i} \in \Omega_{s}} \alpha_{i} e_{i}^{2} /\|e\|_{P_{x}}^{2}
$$

To proceed, lets assume that standard SAT's have been used in the time interval $t \in\left[0, t_{0}\right]$, and that the MPT is applied in the interval $t \in\left[t_{0}, T\right]$, where $T$ is the total simulation time. In the first interval, where only standard SAT's are used, $\eta(t)=0$ and (9) leads to,

$$
\|e\|_{P_{x}} \leq\|e(0)\|_{P_{x}}+t\|T e\|_{P_{x}}^{\max }, \quad t \in\left[0, t_{0}\right],
$$

i.e. the error grows linearly in time.

Next, we consider the interval $t \in\left[t_{0}, T\right]$ where the MPT is applied. In this interval, we may assume that $\eta(t) \geq \eta_{0}>0$, where $\eta_{0}$ is a constant, to obtain the estimate,

$$
\|e\|_{P_{x}} \leq e^{-\eta_{0}\left(t-t_{0}\right)}\left\|e\left(t_{0}\right)\right\|_{P_{x}}+\frac{1-e^{-\eta_{0}\left(t-t_{0}\right)}}{\eta_{0}}\|T e\|_{P_{x}}^{\max }, \quad t \in\left[t_{0}, T\right] .
$$

Consequently, the error decays exponentially to a constant level and continues to stay there when the MPT is applied. For a detailed analysis of error bounded schemes, where it is shown that $\eta_{0}>0$, see [12].

Remark 1. In many applications, exact data is not available. Instead, one have to use data with a certain amount of error. Hence, the method should only be used when the added external data is more accurate than the computed numerical solution at a certain time level. This is typically the case after long time calculations. The effect of inaccurate data is investigated in Appendix A. 


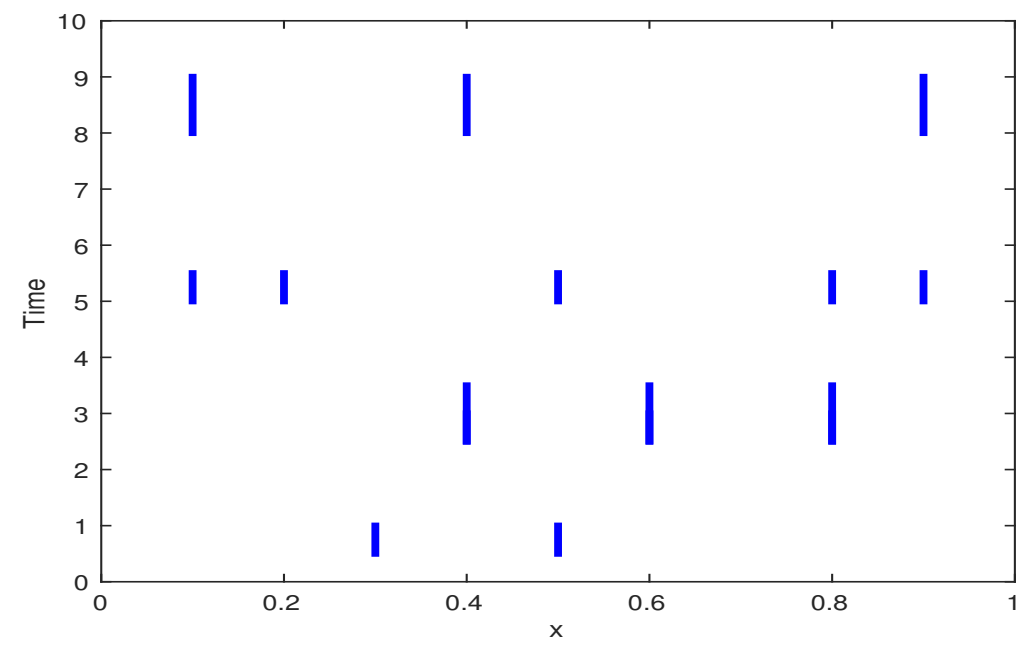

Figure 1: An illustration how the MPT is applied. The blue lines represent the grid points in time and space where the MPT is applied.

\subsection{Initial numerical results}

Consider the numerical scheme (3) with $\alpha_{x}=-\beta_{x}=-1 / 2$ and $\alpha_{i j}=$ -1 . Let the data $\bar{f}$ and $\bar{g}_{i j}$ be extracted from the exact solution $u(x, t)=$ $\sin 2 \pi(x-t)$. SBP operators with third order overall accuracy is used to approximate the derivatives, and the simulation time is $T=30$. When applying the MPT, we choose a set of time intervals. In each time window, we choose $N_{M P T}=10$ spatial grid points at random positions, where additional penalties are applied; see Figure 1 for an illustration. The temporal and spatial grid spacings are $\Delta t=1 / 100$ and $\Delta x=1 / 40$, respectively. In each time interval, the number of spatial grid points where the MPT is applied may vary. We solve the system for three cases with the MPT applied at the time intervals $\Omega_{T, 1}=\{t \in[5,7] \cup[15,17] \cup[25,27]\}, \Omega_{T, 2}=\{t \in[5,10] \cup$ $[15,20] \cup[25,30]\}$ and $\Omega_{T, 3}=\{t \in[5,7] \cup[10,12] \cup[15,17] \cup[20,22] \cup[25,27]\}$.

In Figure 2, the error as a function of time is displayed. One can see that the error grows linearly in time if the standard SAT's are used, as predicted by (11). By using the MPT in time, the error is reduced and kept at a constant level, as indicated by 12 .

To clarify the results above, consider the case where the MPT is applied at the time interval $\Omega_{T, 1}=\{t \in[5,7] \cup[15,17] \cup[25,27]\}$. In Figure 3 , 


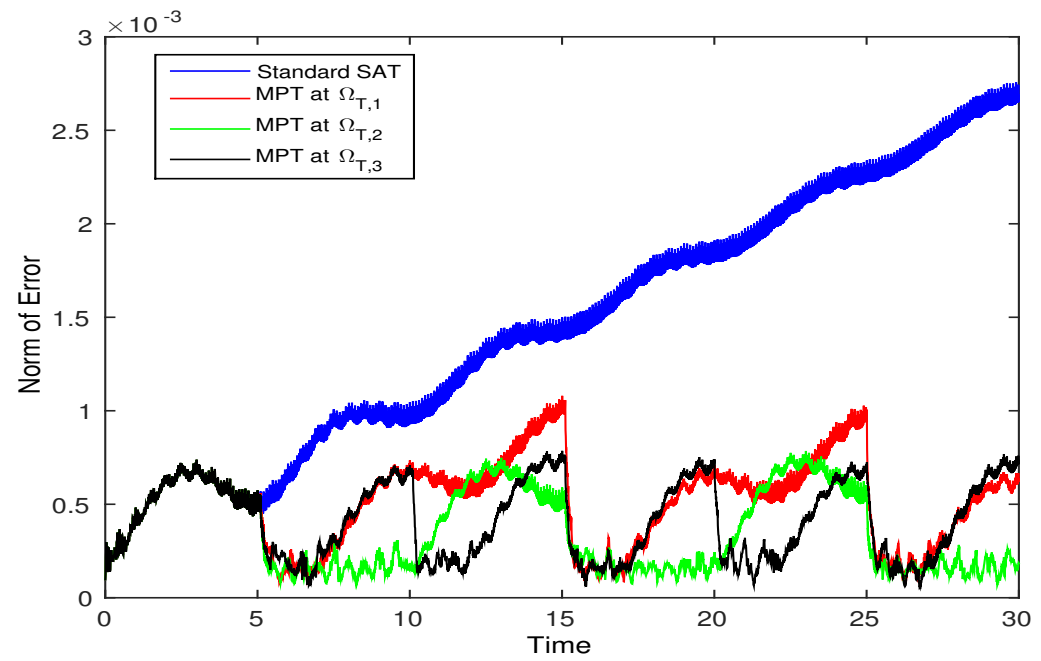

Figure 2: The error as a function of time using standard SAT's and the MPT in time. $\Omega_{T, 1}=\{t \in[5,7] \cup[15,17] \cup[25,27]\}, \Omega_{T, 2}=\{t \in[5,10] \cup[15,20] \cup[25,30]\}$ and $\Omega_{T, 3}=$ $\{t \in[5,7] \cup[10,12] \cup[15,17] \cup[20,22] \cup[25,27]\} . N_{M P T}=10$.

both the error and the function $\eta(t)$, given by (10), is displayed. As one can see, $\eta$ becomes rather large when the MPT is applied, resulting in a rapid exponential decay of error. In Figure 4, a close up around $t=15$ of Figure 3 is shown. One can clearly see the exponential decay of the error.

In conclusion, one can prevent the error growth by implementing observed data using the MPT. Figure 2 also show that the effect is more pronounced when making longer or more frequent observations.

\section{The MPT in time and space for the shallow water equations}

As a more realistic application, we consider the linearized shallow water equations with periodic boundary conditions,

$$
\begin{aligned}
u_{t}+A u_{x}+B u_{y}+C u & =0, \quad(x, y) \in[0,1], t \in[0, T] \\
u(0, y, t) & =u(1, y, t), \\
u(x, 0, t) & =u(x, 1, t), \\
u(x, y, 0) & =f(x, y),
\end{aligned}
$$



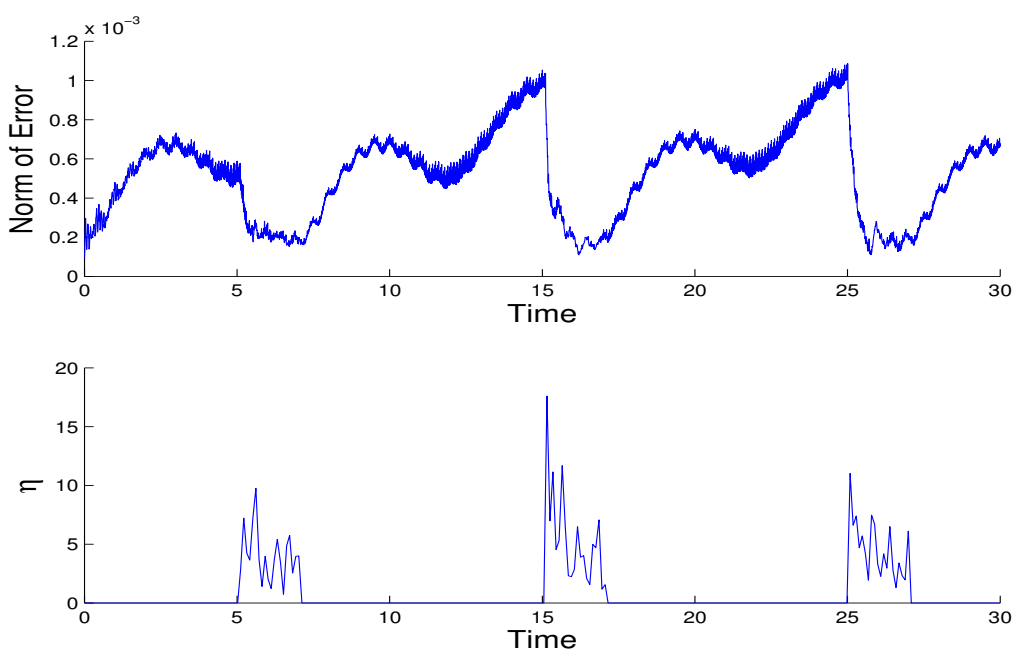

Figure 3: The error as a function of time using the MPT in the interval $\Omega_{T, 1}=\{t \in$ $[5,7] \cup[15,17] \cup[25,27]\}$ (upper figure), and the corresponding function $\eta(t)$ (lower figure).
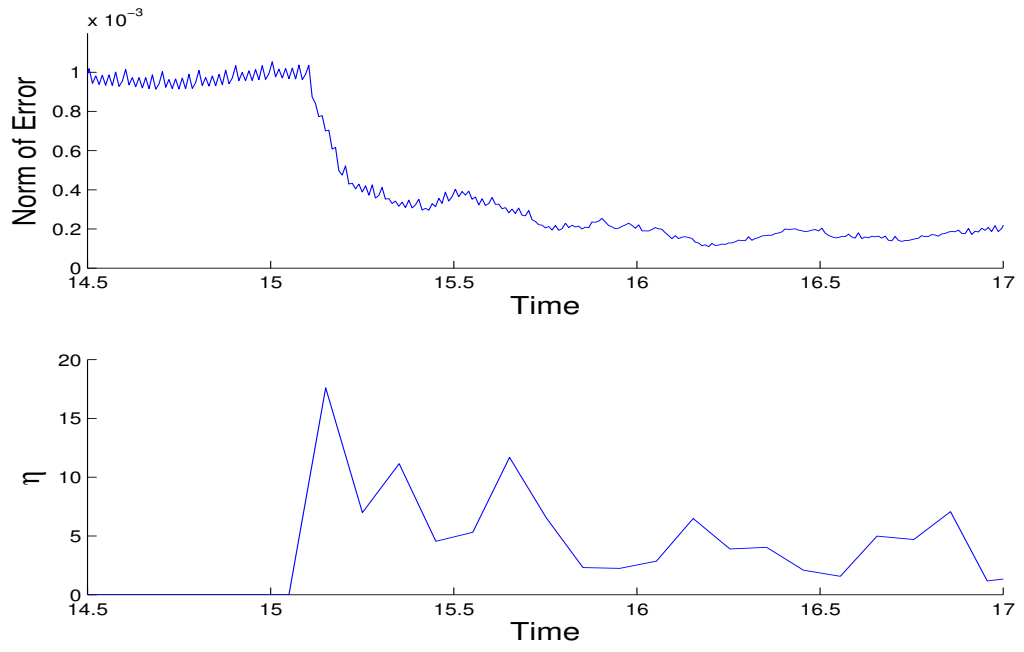

Figure 4: A close up of the error decay and the function $\eta(t)$ around $t=15$ of Figure 3. 
where $f(x, y)$ is the initial data. The matrices $A, B$ and $C$ are defined as

$$
A=\left[\begin{array}{ccc}
\bar{u} & 0 & \bar{c} \\
0 & \bar{u} & 0 \\
\bar{c} & 0 & \bar{u}
\end{array}\right], \quad B=\left[\begin{array}{ccc}
\bar{v} & 0 & 0 \\
0 & \bar{v} & \bar{c} \\
0 & \bar{c} & \bar{v}
\end{array}\right], \quad C=\left[\begin{array}{ccc}
0 & f_{C} & 0 \\
f_{C} & 0 & 0 \\
0 & 0 & 0
\end{array}\right],
$$

in which $\bar{u}$ and $\bar{v}$ are the reference state velocities, $f_{C}$ is the Coriolis parameter and $\bar{c}$ is the gravity wave speed. The vector of variables is $u=\left[u^{\prime}, v^{\prime}, g h^{\prime} / \bar{c}\right]^{T}$, where $u^{\prime}, v^{\prime}$ are the deviations from the reference state velocities $\bar{u}, \bar{v}$ respectively, $g$ is the gravitational constant and $h^{\prime}$ is the deviation from the reference height. For details on how to linearize the shallow water equations, the reader is referred to [7].

By applying the energy method to (13), i.e. multiplying with $u^{T}$ from the left and integrating in space and time, results in

$$
\int_{0}^{1} \int_{0}^{1}\|u(x, y, T)\|^{2} d x d y=\int_{0}^{1} \int_{0}^{1}\|f\|^{2} d x d y .
$$

Equation (14) implies that the solution is bounded by the initial data, and hence the problem (13) is well-posed.

\subsection{The discrete problem}

Next, (13) is discretized using the SBP-SAT technique. Similar to the one-dimensional case described in Section 2, we assume that additional data $g(x, y, t)$ is available at a number of points in space and time. As before, we denote the set of these points $\Omega_{s}$. The discrete scheme that approximates (13) is then,

$$
\begin{array}{r}
\left(D_{t} \otimes I_{x} \otimes I_{y} \otimes I\right) v+\left(I_{t} \otimes D_{x} \otimes I_{y} \otimes A\right) v+\left(I_{t} \otimes I_{x} \otimes D_{y} \otimes B\right) v+ \\
\left(I_{t} \otimes I_{x} \otimes I_{y} \otimes C\right) v=\alpha_{x}\left(I_{t} \otimes P_{x}^{-1} E_{0 x} \otimes I_{y} \otimes A\right)\left(v_{x=0}-v_{x=1}\right)+ \\
\beta_{x}\left(I_{t} \otimes P_{x}^{-1} E_{N x} \otimes I_{y} \otimes A\right)\left(v_{x=1}-v_{x=0}\right)+ \\
\alpha_{y}\left(I_{t} \otimes I_{x} \otimes P_{y}^{-1} E_{0 y} \otimes B\right)\left(v_{y=0}-v_{y=1}\right)+ \\
\beta_{y}\left(I_{t} \otimes I_{x} \otimes P_{y}^{-1} E_{N y} \otimes B\right)\left(v_{y=1}-v_{y=0}\right)+ \\
\alpha_{t}\left(P_{t}^{-1} E_{0 t} \otimes I_{x} \otimes I_{y} \otimes I\right)\left(v_{t=0}-\bar{f}\right)+ \\
\sum_{x_{i}, y_{j}, t_{k} \in \Omega_{s}} \alpha_{i j k}\left(P_{t}^{-1} E_{t k} \otimes P_{x}^{-1} E_{x i} \otimes P_{y}^{-1} E_{y j} \otimes I\right)\left(v_{i j k}-\bar{g}_{i j k}\right),
\end{array}
$$

where $\bar{f}, \bar{g}_{i j k}$ are data injected at the appropriate grid points, $v_{i j k}=\left(E_{t i} \otimes\right.$ $\left.E_{x j} \otimes E_{y k} \otimes I\right) v$ and $\alpha_{x, y}, \beta_{x, y}, \alpha_{t}, \alpha_{i j k}$ are penalty coefficients to be determined. 
The summation on the right hand side runs over all temporal and spatial grid points inside $\Omega_{s}$.

Applying the discrete energy method (multiplying with $v^{T}\left(P_{t} \otimes P_{x} \otimes P_{y} \otimes I\right)$ from the left and adding the transpose of the outcome) to 15 yields,

$$
\begin{array}{r}
v^{T}\left(E_{N t} \otimes P_{x} \otimes P_{y} \otimes I\right) v=\bar{f}^{T}\left(E_{0 t} \otimes P_{x} \otimes P_{y} \otimes I\right) \bar{f}- \\
\left(v_{t=0}-\bar{f}\right)^{T}\left(E_{0 t} \otimes P_{x} \otimes P_{y} \otimes I\right)\left(v_{t=0}-\bar{f}\right)+ \\
\sum_{x_{i}, y_{j}, t_{k} \in \Omega_{s}} \alpha_{i j}\left|v_{i j k}-\bar{g}_{i j k}\right|^{2}+\alpha_{i j k}\left(\left|v_{i j k}\right|^{2}-\left|\bar{g}_{i j k}\right|^{2}\right)
\end{array}
$$

where we have used $\alpha_{x}=\alpha_{y}=-\beta_{x}=-\beta_{y}=-1 / 2$ and $\alpha_{t}=-1$. With the choices $\alpha_{i j} \leq 0$, the solution is bounded by data, and the scheme is stable. Moreover, if $v_{t=0}=\bar{f}$ and $v_{i j k}=\bar{g}_{i j k}$, then (16) mimics the continous energy estimate (14). The MPT adds on a dissipative term, which will lead to an error bound, just as in the one-dimensional case.

\subsection{Error analysis}

The semi-discrete error equation corresponding to 15 is,

$$
\begin{array}{r}
e_{t}+\left(D_{x} \otimes I_{y} \otimes A\right) e+\left(I_{x} \otimes D_{y} \otimes B\right) e+\left(I_{x} \otimes I_{y} \otimes C\right) e= \\
\frac{1}{2}\left(P_{x}^{-1} E_{0 x} \otimes I_{y} \otimes A\right)\left(e_{x=0}-e_{x=1}\right)-\frac{1}{2}\left(P_{x}^{-1} E_{N x} \otimes I_{y} \otimes A\right)\left(e_{x=1}-e_{x=0}\right)+ \\
\frac{1}{2}\left(I_{x} \otimes P_{y}^{-1} E_{0 y} \otimes B\right)\left(e_{y=0}-e_{y=1}\right)-\frac{1}{2}\left(I_{x} \otimes P_{y}^{-1} E_{N y} \otimes B\right)\left(e_{y=1}-e_{y=0}\right)+ \\
\sum_{x_{i}, y_{j}, t \in \Omega_{s}} \alpha_{i j}\left(P_{x}^{-1} E_{x i} \otimes P_{y}^{-1} E_{y j} \otimes I\right) e_{i j}+T e
\end{array}
$$

where $T e$ is the truncation error. Multiplying (17) with $e^{T}\left(P_{x} \otimes P_{y}\right)$ from the left and adding the transpose of the outcome results in,

$$
\frac{\partial}{\partial t}\left(\|e\|_{P_{x} \otimes P_{y}}\right) \leq-\hat{\eta}(t)\|e\|_{P_{x} \otimes P_{y}}+\|T e\|_{P_{x} \otimes P_{y}}^{\max },
$$

where, similar to the model problem,

$$
\hat{\eta}(t)=\sum_{x_{i}, y_{j}, t \in \Omega_{s}} e_{i j}^{2} /\|e\|_{P_{x}}^{2}
$$

In (18), we have chosen $\alpha_{i j}=-1$. 
As in Section 2.2, we assume that standard SAT's are used in the time interval $t \in\left[0, t_{0}\right]$, such that $\hat{\eta}(t)=0$, and that the MPT is applied at the time interval $t \in\left[t_{0}, T\right]$. The solution to (18) then becomes,

$$
\begin{aligned}
& \|e\|_{P_{x} \otimes P_{y}} \leq\|e(0)\|_{P_{x} \otimes P_{y}}+t\|T e\|_{P_{x} \otimes P_{y}}^{\max }, \quad t \in\left[0, t_{0}\right] \\
& \|e\|_{P_{x} \otimes P_{y}} \leq e^{-\hat{\eta}_{0}\left(t-t_{0}\right)}\left\|e\left(t_{0}\right)\right\|_{P_{x} \otimes P_{y}}+\frac{1-e^{-\hat{\eta}_{0}\left(t-t_{0}\right)}}{\hat{\eta}_{0}}\|T e\|_{P_{x} \otimes P_{y}}^{\max }, \quad t \in\left[t_{0}, T\right],
\end{aligned}
$$

where we have assumed that $\hat{\eta}(t) \geq \hat{\eta}_{0}>0$, where $\hat{\eta}_{0}$ is a constant. As one can see, the error grows linearly in time without MPT, and decays exponentially to a maximum level when it is applied.

Remark 2. Note that the denominator of $\hat{\eta}(t)$ scales with $\Delta x$, and that the numerator does not. Consequently, if the MPT is applied at many spatial grid points, $\hat{\eta}$ will become large, resulting in a rapid decay to low error levels.

\subsection{Numerical results}

Consider the numerical scheme (15) with $\bar{u}=\bar{v}=\bar{c} / 2=1$ and the penalty terms $\alpha_{x}=\alpha_{y}=-\beta_{x}=-\beta_{y}=1 / 2$ and $\alpha_{i j k}=-1$. Again, we use an SBP scheme of third order overall accuracy with the grid parameters $\Delta x=\Delta y=1 / 20$ and $\Delta t=1 / 100$. The simulation time is $T=30$ and additional penalties are applied in the time intervals $\Omega_{T, 1}=\{t \in[5,7] \cup$ $[15,17] \cup[25,27]\}, \Omega_{T, 2}=\{t \in[5,10] \cup[15,20] \cup[25,30]\}$ and $\Omega_{T, 3}=\{t \in$ $[5,7] \cup[10,12] \cup[15,17] \cup[20,22] \cup[25,27]\}$. The method of manufactured solutions [9] is applied to (13) by adding a forcing function to the right-hand side, such that the exact solution becomes $u=\sin (2 \pi(x+y-2 t))[1,-1,0]^{T}$.

In Figure 5, the error as a function of time is shown when the MPT is applied at $N_{M P T}=50$ spatial grid points in each time window. The MPT prevents the error from growing, just as in the one-dimensional case previously discussed.

Next, we consider the case where the MPT is applied during very short time intervals. In Figure 6, the MPT is applied at the time intervals $\Omega_{T, 1}=$ $\{t \in[5,5.1] \cup[15,15.1] \cup[25,25.1]\}$ and $\Omega_{T, 2}=\{t \in[5,6] \cup[15,16] \cup[25,26]\}$. As one can see, the error reduction is significant even when the observations are made during short time spans.

In the examples a above, the MPT is applied at a significant number of spatial grid points in each time interval, resulting in a rapid decay of error and small error bounds. Next, we study how the error behaves when the MPT is applied only at a few spatial grid points. 


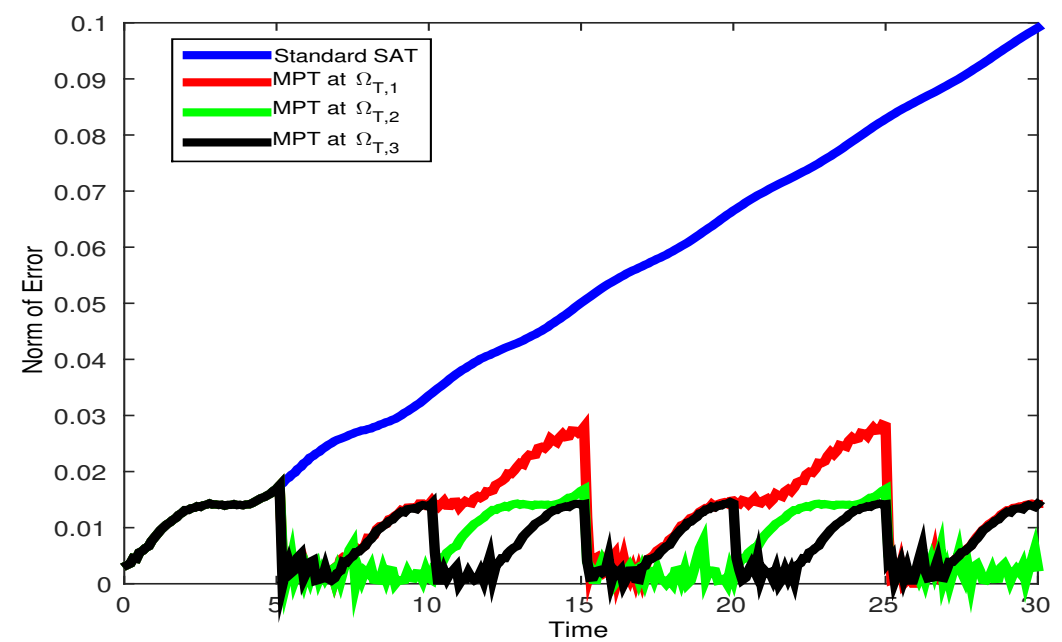

Figure 5: The error as a function of time using standard SAT's and the MPT in time. $\Omega_{T, 1}=\{t \in[5,7] \cup[15,17] \cup[25,27]\}, \Omega_{T, 2}=\{t \in[5,10] \cup[15,20] \cup[25,30]\}$ and $\Omega_{T, 3}=$ $\{t \in[5,7] \cup[10,12] \cup[15,17] \cup[20,22] \cup[25,27]\} . N_{M P T}=50$.

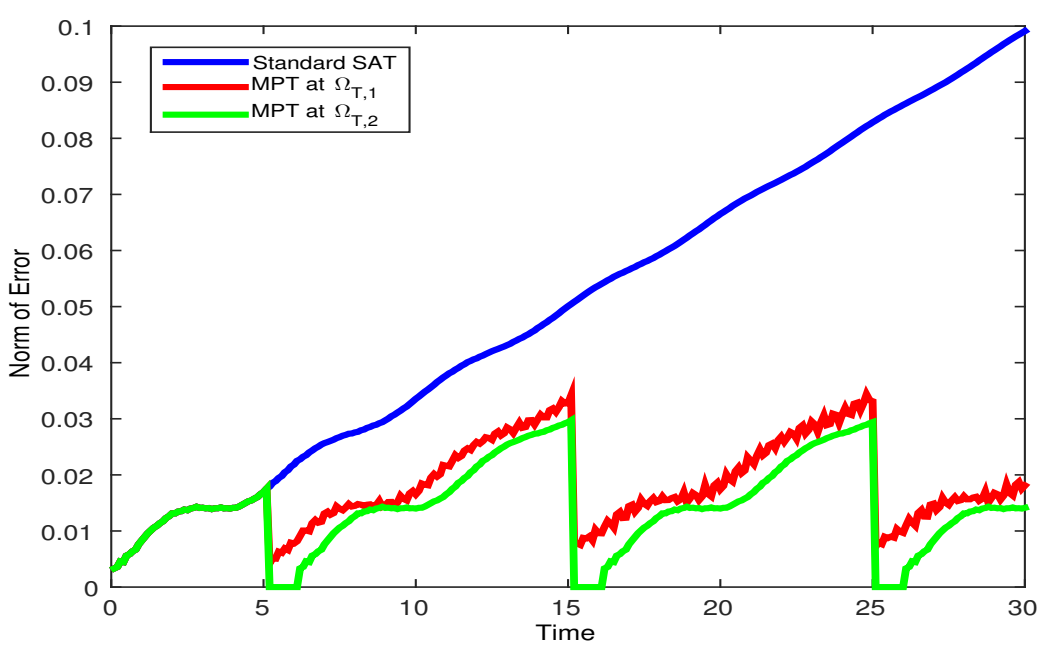

Figure 6: The error as a function of time using standard SAT's and the MPT in time. $\Omega_{T, 1}=\{t \in[5,5.1] \cup[15,15.1] \cup[25,25.1]\}, \Omega_{T, 2}=\{t \in[5,6] \cup[15,16] \cup[25,26]\} . N_{M P T}=$ 50 . 


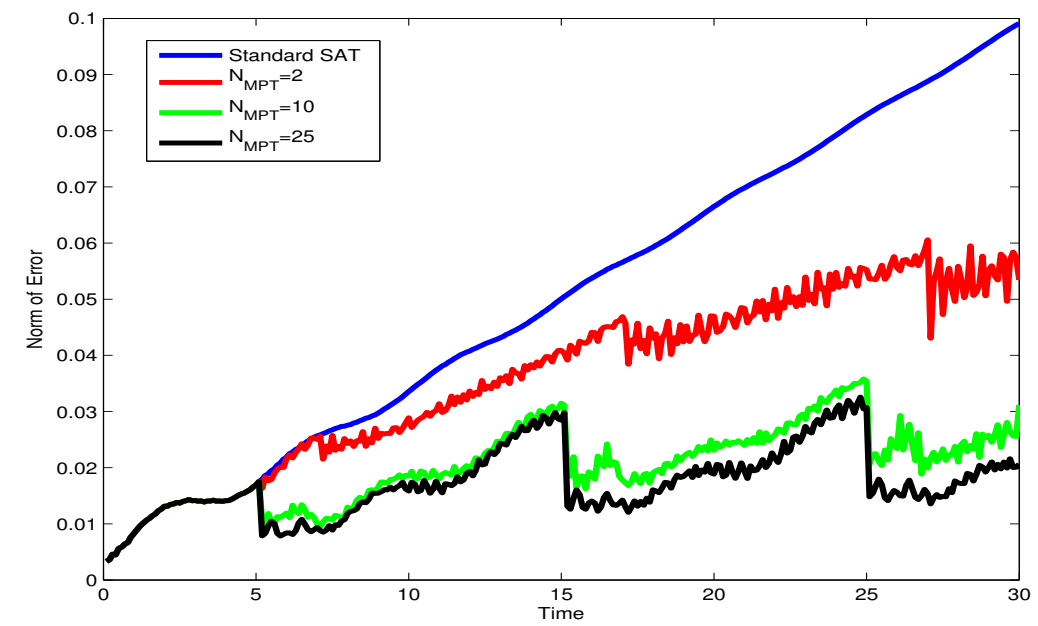

Figure 7: The error as a function of time using standard SAT's and the MPT in time. $N_{M P T}$ additional penalty terms are applied in the time interval $\Omega_{T, 1}=\{t \in[5,7] \cup$ $[15,17] \cup[25,27]\}$.

In Figure 7, additional penalties are applied in the time intervals $\Omega_{T, 1}=$ $\{t \in[5,7] \cup[15,17] \cup[25,27]\}$ at $N_{M P T}=2,10,25$ grid points in space. As expected, the effect is more pronounced when the MPT is applied at many spatial grid points. However, one can observe a notable reduction in the error growth even when the MPT is applied at very few spatial grid points.

\section{Summary and conclusions}

The MPT technique introduced in [13] has been extended such that it can be applied at limited time intervals, to model real life observations better. The resulting numerical scheme is proven to be unconditionally stable with appropriate choices of penalty parameters.

The MPT terms adds on a dissipative term in the energy estimate, which result in an error bound. When the MPT is applied, the error will decay exponentially until it reaches a constant level.

Numerical experiments has been performed on the advection equation and the linearized shallow water equations, and the results show that one can limit or prevent the error growth for long simulations by using the MPT 
in both time and space. The effect is significant even when data is available at very few spatial grid points, and during very short observation time.

\section{Appendix A. The MPT with inaccurate data}

In order to explain the effect of inaccurate external data, consider the error equation (7) with perturbed data,

$$
\begin{array}{r}
e_{t}+D_{x} e=-\frac{1}{2} P_{x}^{-1} E_{0 x}\left(e_{0}-e_{N}\right)+\frac{1}{2} P_{x}^{-1} E_{N x}\left(e_{N}-e_{0}\right)+ \\
\sum_{x_{i}, t \in \Omega_{s}} \alpha_{i} P_{x}^{-1} E_{j x}\left(e_{i}-\Delta g_{i}\right)+T e
\end{array}
$$

where $\Delta g_{i}$ denotes a small peturbation in the data. Applying the energy method to (A.1) and performing a similar analysis as in Section 2.2 results in,

$$
\frac{\partial}{\partial t}\left(\|e\|_{P_{x}}\right) \leq-\eta(t)\|e\|_{P_{x}}+\sum_{x_{i}, t \in \Omega_{s}}\left|\alpha_{i}\right| \frac{\left|e_{i}\right|}{\|e\|_{P}}\left|\Delta g_{i}\right|+\|T e\|_{P_{x}}^{\max }
$$

where we have used that $\alpha_{i} \leq 0$ and

$$
\eta(t)=-\sum_{x_{i} \in \Omega_{s}} \alpha_{i} e_{i}^{2} /\|e\|_{P_{x}}^{2}
$$

First, consider the case where there is an upper limit of the ratio $\left|e_{i}\right| /\|e\|_{P_{x}}$, i.e. $\left|e_{i}\right| /\|e\|_{P_{x}} \leq \beta_{0}$ for some constant $\beta_{0}$. In this case, we can easily show that the error is bounded according to

$$
\|e\|_{P_{x}} \leq \frac{1}{\eta_{0}}\left(\|T e\|_{P_{x}}^{\max }+\beta_{0} \sum_{x_{i}, t \in \Omega_{s}}\left|\alpha_{i} \| \Delta g\right|_{\max }\right)
$$

where $|\Delta g|_{\text {max }}$ is an upper estimate of $\left|\Delta g_{i}\right|$. Note that $|\Delta g|_{\text {max }}$ is small compared to $\left|e_{i}\right|$ since otherwise we would not impose the additional penalties. Note also that $\sum_{x_{i}, t \in \Omega_{s}}\left|\alpha_{i}\right|$ is bounded.

Next, we consider the case where the ratio $\left|e_{i}\right| /\|e\|_{P_{x}}$ is arbitrary large. We may then assume that

$$
\sum_{x_{i}, t \in \Omega_{s}}\left|\alpha_{i}\left\|e_{i} \mid /\right\| e \|_{P_{x}} \leq \eta(t)\right.
$$


Using A.4 in (A.2) results in,

$$
\frac{\partial}{\partial t}\left(\|e\|_{P_{x}}\right) \leq-\eta(t)\|e\|_{P_{x}}+\eta(t)|\Delta g|_{\max }+\|T e\|_{P_{x}} .
$$

Solving A.5 for $\|e\|_{P_{x}}$ yields,

$$
\|e\|_{P_{x}} \leq\left(1-e^{-H(t)}\right)|\Delta g|_{\max }+e^{-H(t)} \int_{0}^{t} e^{H(\tau)}\|T e\|_{P_{x}} d \tau,
$$

where $H(t)=\int_{0}^{t} \eta(\tau) d \tau$ is non-negative.

From previous analysis in Section 2.2, we know that the second term on the right-hand side of (A.6) is bounded. Hence, we can conclude that the error is bounded by $\|T e\|_{P_{x}}$ and $|\Delta g|_{\max }$.

\section{References}

[1] R. Anthes. Data assimilation and initialization of hurricane prediction models. Journal of the Atmospheric Sciencies, 31:702-719, 1973.

[2] M. Carpenter, D. Gottlieb, and S. Abarbanel. Time-stable boundary conditions for finite-difference schemes solving hyperbolic systems: methodology and application to high-order compact schemes. Journal of Computational Physics, 111:220-236, 1994.

[3] M. Carpenter, J. Nordström, and D. Gottlieb. A stable and conservative interface treatment of arbitrary spatial accuracy. Journal of Computational Physics, 148:341-365, 1999.

[4] P. Courtier, E. Andersson, W. Heckley, D. Vasiljevic, M. Hamrud, F. Rabier, M. Fisher, and J. Pailleux. The ECMWF implementation of threedimensional variational assimilation (3D-Var) I: formulation. Quarterly Journal of the Royal Meteorological Society, 124:1783-1807, 1998.

[5] J. Derber and F. Bouttier. A reformulation of the background error covariance in the ECMWF global data assimilation system. Tellus, 51:195$221,1999$.

[6] H. Frenander and J. Nordström. A stable and accurate Davies-like relaxation procedure using multiple penalty terms for lateral boundary conditions. Dynamics of Atmospheres and Oceans, 73:34-46, 2016. 
[7] S Ghader and J Nordström. Revisiting well-posed boundary conditions for the shallow water equations. Dynamics of Atmospheres and Oceans, 66:1-9, 2014.

[8] B. Gustafsson, H. Kreiss, and A. Sundström. Stability theory of difference approximations for mixed initial boundary value problems II. Mathematics of Computation, 26:649-686, 1972.

[9] J. Lindström and J. Nordström. A stable and high-order accurate conjugate heat transfer problem. Journal of Computational Physics, 229:5440-5456, 2010.

[10] T. Lundquist and J. Nordström. The SBP-SAT technique for initial value problems. Journal of Computational Physics, 270:86-104, 2014.

[11] K. Mattsson and J. Nordström. Summation by parts operators for finite difference approximations of second derivatives. Journal of Computational Physics, 199:503-540, 2004.

[12] J. Nordström. Error bounded schemes for time-dependent hyperbolic problems. SIAM Journal of Scientific Computing, 30:46-59, 2007.

[13] J. Nordström, Q. Abbas, B.A. Erickson, and H. Frenander. A flexible boundary procudure for hyperbolic problems: multiple penalty terms applied in a domain. Communications in Computational Physics, 16:541-570, 2014.

[14] J. Nordström and T. Lundquist. Summation-by-parts in time. Journal of Computational Physics, 251:487-499, 2013.

[15] D. Stauffer and N. Seaman. Use of four-dimensional data assimilation in a limited-area mesoscale model. Part I: experiments with synoptic-scale data. Monthly Weather Review, 110:1250-1277, 1990.

[16] B. Strand. Summation by parts for finite difference approximations for $\frac{d}{d x}$. Journal of Computational Physics, 110:47-67, 1994.

[17] M. Svärd and J. Nordström. Review of summation-by-parts schemes for initial-boundary-value problems. Journal of Computational Physics, 268:17-38, 2014. 